\title{
Socioeconomic Indices Performance for HIV Diagnostic on Severely Malnourished Children in Burkina Faso
}

\author{
Léon G. Blaise Savadogo ${ }^{1,2 *}$, Philippe Donnen ${ }^{3}$, Michèle Dramaix ${ }^{2}$ \\ ${ }^{1}$ Department of Epidemiology and Public Health, Child and Mother Health, Nutrition and Survival Unit, \\ Institut Supérieur des Sciences de la Santé, Bobo Dioulasso, Burkina Faso \\ ${ }^{2}$ CR2-Epidemiology-Biostatistics-Clinical Research, School of Public Health, Université Libre de Bruxelles, \\ Bruxelles, Belgique \\ ${ }^{3}$ CR3-Politiques et Systèmes de Santé, Santé Internationale, School of Public Health, Université Libre de \\ Bruxelles, Bruxelles, Belgique \\ Email: "gueswende@hotmail.com
}

Received 10 February 2014; revised 10 March 2014; accepted 17 March 2014

Copyright (C) 2014 by authors and Scientific Research Publishing Inc.

This work is licensed under the Creative Commons Attribution International License (CC BY).

http://creativecommons.org/licenses/by/4.0/

(c) (i) Open Access

\begin{abstract}
Children living with HIV should be diagnosed as early as possible and linked to antiretroviral treatment. The costs of HIV virological testing, individual and social implications of the disease require critical quality of the medical approach to diagnostic strategy. Socioeconomic data can be used to refine the medical decision. The objective of this analysis was to determine informative capacity of socioeconomic indices in the diagnosis and approach of HIV in severely malnourished children using receiver operating characteristic (ROC) curves. Materials and Methods: This was a test performance analysis conducted from data of severely malnourished children at an inpatient feeding therapeutic center in Ouagadougou. Anthropometric and social indices have been used as tests; their sensitivity, specificity and predictive values have been calculated. ROC curves and areas under curves were obtained. Medical decision aid scheme has been validated from severely malnourished children data. Results: Socioeconomic indices showed high performance to discriminate severely malnourished HIV infected and uninfected children, with high area under ROC curves. The medical decision aid scheme specificity reached $97.6 \%$ with predictive values close to 85\%. Conclusion: This approach can help for expanding ART for children in low income countries and achieve the maximum ART benefits in child socioeconomic kneed context.
\end{abstract}

\section{Keywords}

Socioeconomic Indices; Test Performance; HIV; Severely Malnourished Children

\footnotetext{
"Corresponding author.
} 


\section{Introduction}

Despite international and in-country initiatives to accelerate elimination of mother-to-child HIV transmission, percentage of pregnant women who receive HIV testing and percentage of pregnant women living with HIV receiving ARV medicines for preventing mother-to-child transmission (PMTCT), remain very low in many low income countries, with a range of less than $37 \%$ to less than $48 \%$ (20\% in Burkina Faso) [1]. In these countries, very few HIV-exposed infants have access to early diagnosis and to "timely" initiation of antiretroviral therapy (ART). Currently among HIV-exposed infants needing testing, only an estimated $15 \%$ are tested in the first two months of life [2]. The number of children receiving ART in low income countries increased in 2012 but, in West and Central Africa, ART coverage of children remain very low, less than $13 \%$ [2]. There is an urgent need to renew efforts for expanding ART for children to achieve the maximum ART benefits. There is a need to combine several approaches to identify greater numbers of children living with HIV. Many studies reported high HIV prevalence, more than 29\% among severely malnourished children in sub-Saharan Africa [3]-[7]. Children living with HIV should be diagnosed as early as possible and linked to the treatment program to maximize ART benefits. It is strongly recommended that HIV serological assays be used as a screening assay to determine HIV exposure at less than 18 months of age and as a diagnostic assay at up 18 months of age; and it is strongly recommended that HIV virological testing be used to diagnose HIV infection in infants and children below 18 months of age. In infants and children undergoing virological testing, the following assays are strongly recommended for use: HIV DNA on whole blood specimen or dried blood spot (DBS); HIV RNA on plasma or DBS; and p24 Ag on plasma or DBS [8]. The costs of HIV virological testing, individual and social implications of the disease, request request from care providers a critical quality of the medical approach to diagnostic. Diagnostic approach is important for therapeutic success. At the contact point with a child, its socioeconomic data are the first sources of information before clinical and biological data. The information can be used to refine the medical decision for the purpose of HIV diagnosis. Diagnostic performance of a test is the sensitivity, specificity and predictive values. The objective of this analysis was to determine the informative capacity of socioeconomic indices in the diagnostic approach of HIV in severely malnourished children using ROC curves.

\section{Materials and Methods}

\subsection{Study Design and Sites}

This was a test performance analysis conducted from data of severely malnourished children hospitalized, between 1999 and 2005, at an inpatient feeding therapeutic center in Ouagadougou.

\subsection{Study Population}

The subjects were from under five old hospitalized severe acute (SAM) malnourished children. SAM diagnostic was based on severe wasting, as a weight-for-height below -3 SD or below $70 \%$ of median (NCHS reference) or the presence of nutritional oedema affecting both feet. All children include in the analysis were HIV status naive at admission. After admission, severely malnourished children's parents (mothers and father) consent was acquired for HIV diagnosis. When parents provided informed consent for HIV testing, the child's blood sample was collected and identified by a single code and immediately transferred to laboratory for analyses. HIV virological testing was not easily accessible in our set-up.

\subsection{HIV Diagnosis}

After admission, severely malnourished children parents (mothers and father) consent was required for HIV diagnosis. When parents provided informed consent for HIV testing, the child blood sample were collected and identified by a single code and immediately transferred to Ouagadougou pediatric hospital laboratory for analyses. Severely malnourished children 18 months or older have HIV serological testing performed according to the standard diagnostic HIV serological testing algorithm used. To reduce testing cost, for malnourished children below 18 months of age, a first level antibody tests (serological testing) was used and polymerase chain reaction (PCR) test was used when antibody test was positive.

\subsection{Study Sample Size}

Data of 481 severely malnourished children, tested for HIV have been included for analysis. 


\subsection{Data Collection}

Data were collected on admission by trained research nurses. Four social characteristics were considered: the death of one or both parents (father and/or mother); severe malnutrition relapse (re-admission for severe malnutrition); the child's parents' socioeconomic level (parental occupation and economic source were used, and two categories were established: low socioeconomic level and medium to high socioeconomic level); and the number of living children in the household. These variables were not part of the center admission criteria's or of the HIV/AIDS diagnosis criteria's, avoiding selection bias.

For anthropometry: child age was determined using date of birth recorded from its mother pregnancy card and date of admission. Weight was measured in $\mathrm{kg}$ using recommended scale with a precision of $0.1 \mathrm{~kg}$ (100 g). Length of less than 2 years old child was measured in centimeters using length board (horizontal infantometer) with a precision $0.1 \mathrm{~cm}(1 \mathrm{~mm})$ and height of 2 years or older child able to stand was measured in $\mathrm{cm}$ using height board (vertical stadiometer) with a precision $0.1 \mathrm{~cm}(1 \mathrm{~mm})$. If the child was not able to stand for any reason, length was measured using length board. Oedema of both feet was automatically considered as severely malnourished case. Mid-upper arm circumference (MUAC) $(\mathrm{cm})$ was recorded for children who had completed over 6 months of age. Anthropometrics indices were calculated and expressed on Z-score: weight-for-height (WHZ), weight-for-age (WAZ) and height-for-age (HAZ).

\subsection{Statistical Analyses}

Standard statistical methods were applied. We used Pearson's Chi square test or Fisher's exact test to compare proportions; and Student's independent t-test to compare means. The data were analyzed using SPSS software.

Anthropometric and social indices have been used as tests. The diagnostic performance and quality of each indice were based on its sensitivity (Se), specificity (Sp), predictive values. Sensitivity expresses the conditional probability of a positive "test" if the disease (HIV infection in this case) was present and the specificity is the probability of a negative "test” between severely malnourished children not infected by HIV. Sensitivity and Specificity with their 95\% confidence interval (95\% CI) indicate the intrinsic qualities of each test and positive and negative predictive values express the extrinsic qualities of the test.

Receiver Operating Characteristic (ROC) curves were obtained by plotting the proportion of true positives or Sensitivity of a diagnostic test (in our case it is the proportion of positives test among HIV infected severely malnourished children) against the proportion of false positives (1-Sp) (the proportion of positive test among non HIV infected severely malnourished children). The diagonal line of the graph represents the "chance line”. More the curve is above the diagonal better is the performance of a diagnostic test for discriminating severely malnourished children HIV infection.

ROC curves have been plotted using SPSS software. The area under the curve that reflects the performance of a "test" and its 95\% confidence interval 95\% has been estimated.

For anthropometrics indices, ROC curves were established using -3 DS cutoff for WHZ, HAZ and HAZ, and two cutoffs, 110 and $100 \mathrm{~mm}$ for MUAC. Only socioeconomic variable statistically associated to HIV infection was used for combining on different socioeconomic indices.

In the first stage, four Socioeconomic indices were obtained from four logistic regression models: indice1: child father and/or mother recent death + child severe malnutrition relapse + child parents Socioeconomic level; indice2: child father and/or mother recent death + child severe malnutrition relapse; indice3: child father and/or mother recent death + child parent Socioeconomic level; indice4: child severe malnutrition relapse + child parents Socioeconomic level. Probabilities predicted by logistic model were used to estimate the area under the corresponding ROC curve.

In a second stage, same variables combinations were used to obtain idem four Socioeconomic indices based on a simple addition score using rating “yes" (variable presence) $=1$ and "No" (variable absence) $=0$. Areas under the ROC curves were also estimated and compared with those obtained from logistic regression models. This method allowed the prioritization of socioeconomic indices and determining a cutoff for severely malnourished children classification as positive (“at risk HIV infected”) or negative not ("at risk HIV infected”) at admission.

A medical decision aid scheme to purpose strongly HIV diagnosis to severely malnourished children at admission has been established, data of 211 severely malnourished children have been used for validation. 


\section{Results}

From the 481 severely malnourished, hospitalized children, 16.8\% were HIV infected at admission.

At admission, between severely malnourished HIV infected and uninfected children, anthropometrics indices differed significantly for WHZ $(p=0.004)$, WAZ $(p=0.002)$, MUAC $(p=0.001)$; severe malnutrition relapse $(\mathrm{p}<0.001)$ and child parents socioeconomic level $(<0.001)$ (Table 1$)$.

Based on areas under ROC curves, performance of anthropometrics indices to discriminate severely malnourished HIV infected and uninfected children was low and the proportion of false positives (1-Sp) was high (Table 2). Socioeconomic indices from logistic regression model and from simple addition score showed equivalent high performance to discriminate severely malnourished HIV infected and uninfected children, with high area under ROC curve (Table 2, Figures 1 and 2).

With appropriate cutoff, Socioeconomic indices showed high specificity (low proportions of false positives) (Table 3).

For the validation, the medical decision aid scheme to purpose strongly HIV diagnosis to severely malnourished children at admission built from socioeconomic indices leads to propose strongly HIV diagnosis to 26 (12.32\% ) on 211 severely malnourished children (Figure 3). The specificity of this diagnostic approach reached $97.6 \%$ and the proportion of false positives was low (2.4\%), positive and negative predictive values are close to $85 \%$.

\section{Discussion}

Despite large efforts, many children continue to be infected with HIV and not identified without receiving timely and efficient treatment. Both, community and intra-hospital severe malnourished children management program, offer opportunity to children ART coverage increasing, especially for under five old. Significant number of HIV diagnosis naïve infants is received in these programs. But as showed, anthropometric indices are not significantly discriminative for severely malnourished children HIV infection diagnosis. It has been established that HIV can lead early to a nutritional deficiency [9] [10], but analyzes did not show performance of anthropometric indices to discriminate severely malnourished HIV infected and uninfected children [11]. Socioeconomic indices showed more high performance in discriminating severe malnourished children HIV infected to uninfected children. Combined, these indices could be used in the medical decision to strongly purpose biological

Table 1. Severely malnourished HIV infected and uninfected children age and anthropometrics indices comparison at admission.

\begin{tabular}{|c|c|c|c|}
\hline & HIV infected & $\mathbf{p}$ & HIV uninfected \\
\hline $\begin{array}{l}\text { Age (month) (n) } \\
\quad \text { Mean (SD) }\end{array}$ & $\begin{array}{c}81 \\
17.54(10.21)\end{array}$ & $0.60^{\mathrm{a}}$ & $\begin{array}{c}400 \\
18.20(10.41)\end{array}$ \\
\hline $\begin{array}{l}\text { WHZ (n) } \\
\text { Mean (SD) }\end{array}$ & $\begin{array}{c}78 \\
-3.69(0.68)\end{array}$ & $0.004^{\mathrm{a}}$ & $\begin{array}{c}377 \\
-3.40(0.82)\end{array}$ \\
\hline $\begin{array}{l}\text { WAZ (n) } \\
\text { Mean (SD) }\end{array}$ & $\begin{array}{c}70 \\
-4.50(0.78)\end{array}$ & $0.002^{\mathrm{a}}$ & $\begin{array}{c}347 \\
-4.17(0.81)\end{array}$ \\
\hline $\begin{array}{l}\text { HAZ (n) } \\
\text { Mean (SD) }\end{array}$ & $\begin{array}{c}370 \\
-2.77(1.25)\end{array}$ & $0.16^{\mathrm{a}}$ & $\begin{array}{c}75 \\
-2.99(1.20)\end{array}$ \\
\hline $\begin{array}{l}\text { MUAC }^{\mathrm{b}}(\mathrm{n}) \\
\text { Mean (SD) }\end{array}$ & $\begin{array}{c}74 \\
9.59(1.22)\end{array}$ & $0.001^{\mathrm{a}}$ & $\begin{array}{c}385 \\
10.12(1.27)\end{array}$ \\
\hline $\begin{array}{l}\text { At least child one parent death (n) } \\
\text { Yes }\end{array}$ & $\begin{array}{c}48 \\
35.4 \%\end{array}$ & $<0.001^{\mathrm{c}}$ & $\begin{array}{c}178 \\
9.6 \%\end{array}$ \\
\hline $\begin{array}{c}\text { Severe malnutrition relapse }(\mathrm{n}) \\
\text { Yes }\end{array}$ & $\begin{array}{c}47 \\
44.7 \%\end{array}$ & $<0.001^{\mathrm{c}}$ & $\begin{array}{c}178 \\
6.2 \%\end{array}$ \\
\hline $\begin{array}{l}\text { Child parents Socioeconomic level (n) } \\
\text { Middle to high }\end{array}$ & $\begin{array}{c}45 \\
60.0 \%\end{array}$ & $<0.001^{\mathrm{c}}$ & $\begin{array}{c}165 \\
26.1 \%\end{array}$ \\
\hline $\begin{array}{l}\text { One living child (n) } \\
\text { Yes }\end{array}$ & $\begin{array}{c}48 \\
45.8 \%\end{array}$ & $0.18^{\mathrm{c}}$ & $\begin{array}{c}178 \\
34.8 \%\end{array}$ \\
\hline
\end{tabular}

${ }^{a}$ t test, ${ }^{b} \geq 6$ months old, ${ }^{c} \chi^{2}$ test. 
Table 2. Area under ROC curves for anthropometrics indices and socioeconomic indices.

\begin{tabular}{|c|c|c|c|}
\hline & Area under ROC curve & $\mathrm{SE}^{\mathbf{b}}$ & 95\%CI \\
\hline \multicolumn{4}{|c|}{ Anthropometrics indices } \\
\hline WHZ & 0.595 & 0.033 & $0.530-0.660$ \\
\hline WAZ & 0.622 & 0.037 & $0.549-0.694$ \\
\hline HAZ & 0.556 & 0.036 & $0.486-0.626$ \\
\hline MUAC (cm) & 0.605 & 0.034 & $0.538-0.672$ \\
\hline \multicolumn{4}{|c|}{ Socioeconomic indices(logistic regression) } \\
\hline Child father or mother death (death F/M) & 0.629 & 0.049 & $0.533-0.726$ \\
\hline Indice1 & 0.842 & 0.039 & $0.766-0.918$ \\
\hline Indice2 & 0.780 & 0.043 & $0.695-0.865$ \\
\hline Indice3 & 0.758 & 0.044 & $0.671-0.845$ \\
\hline Indice4 & 0.771 & 0.044 & $0.684-0.857$ \\
\hline \multicolumn{4}{|c|}{ Socioeconomic indices (scored by addition) } \\
\hline Indice1 & 0.822 & 0.039 & $0.745-0.898$ \\
\hline Indice2 & 0.772 & 0.043 & $0.688-0.857$ \\
\hline Indice3 & 0.747 & 0.044 & $0.661-0.833$ \\
\hline Indice4 & 0.758 & 0.044 & $0.672-0.844$ \\
\hline
\end{tabular}

${ }^{\mathrm{a}}$ Variable considered alone; ${ }^{\mathrm{b}} \mathrm{SE}$ = standard Error, indice1: child father and/or mother recent death + child severe malnutrition relapse + child parents' socioeconomic level; indice2: child father and/or mother recent death + child severe malnutrition relapse; indice3: child father and/or mother recent death + child parent socioeconomic level; indice4: child severe malnutrition relapse + child parents' socioeconomic level.

Table 3. Sensitivity (Se), false positive (1-Sp), positive predictive value (VPP) and negative predictive value (NPV) of anthropometric indices and socioeconomic indices at different cutoff.

\begin{tabular}{|c|c|c|c|c|c|}
\hline & & Se (\%) & 1-Sp (\%) & PPV (\%) & NPV (\%) \\
\hline Anthropometrics indices & Cutoff & & & & \\
\hline WHZ & -3 & 87.2 & 69.2 & 20.4 & 92.2 \\
\hline WAZ & -3 & 98.7 & 92.9 & 17.8 & 96.4 \\
\hline \multirow{2}{*}{ HAZ } & $100 \mathrm{~mm}$ & 66.2 & 52.2 & 19.6 & 88.0 \\
\hline & $110 \mathrm{~mm}$ & 90.5 & 83.1 & 17.3 & 90.3 \\
\hline \multicolumn{6}{|c|}{ Socioeconomic indices (scored by addition) } \\
\hline Child father or mother death (death $\mathrm{F} / \mathrm{M})^{\mathrm{a}}$ & & 9.6 & 35.4 & 50.0 & 16.1 \\
\hline Indice1 & 2 & 4.5 & 0.6 & 66.7 & 79.6 \\
\hline Indice2 & 2 & 12.8 & 0.6 & 85.7 & 81.2 \\
\hline Indice3 & 2 & 17.8 & 0.6 & 88.9 & 81.6 \\
\hline Indice4 & 2 & 27.3 & 1.8 & 80.0 & 83.5 \\
\hline
\end{tabular}

${ }^{\mathrm{a}}$ Variable considered alone; ${ }^{\mathrm{b}} \mathrm{SE}$ = standard Error, indice1: child father and/or mother recent death + child severe malnutrition relapse + child parents' socioeconomic level; indice2: child father and/or mother recent death + child severe malnutrition relapse; indice3: child father and/or mother recent death + child parent socioeconomic level; indice4: child severe malnutrition relapse + child parents' socioeconomic level.

test for HIV diagnosis, especially virological testing for less than 18 months old. Used variables for the socioeconomic indices are simple, can be easily obtained, from child parent or guardian. Care givers did not need specific training or material to collect these routinely data. The error risk is limited (no systematic measurement error, reliable source of information). These indices can help to identify most vulnerable children and prepare HIV biological testing process, including positive result announcement process, and context to efficiency ART initia- 


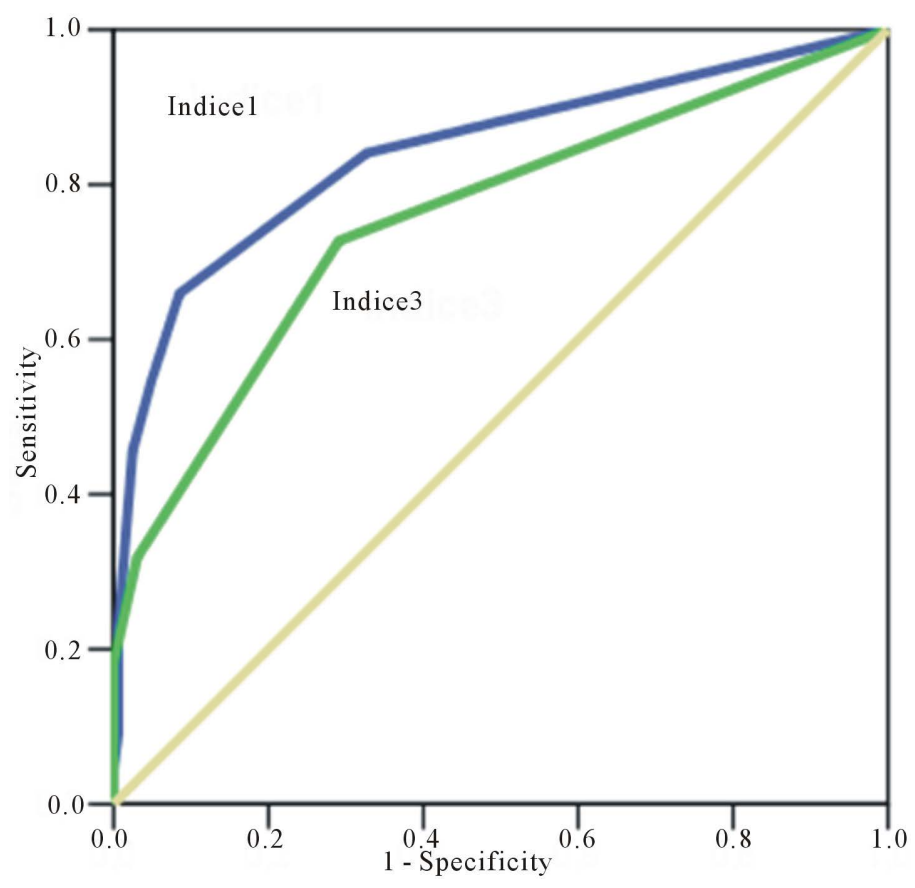

Figure 1. ROC curves for socioeconomic indice1 and indice3 (from logistic regression).

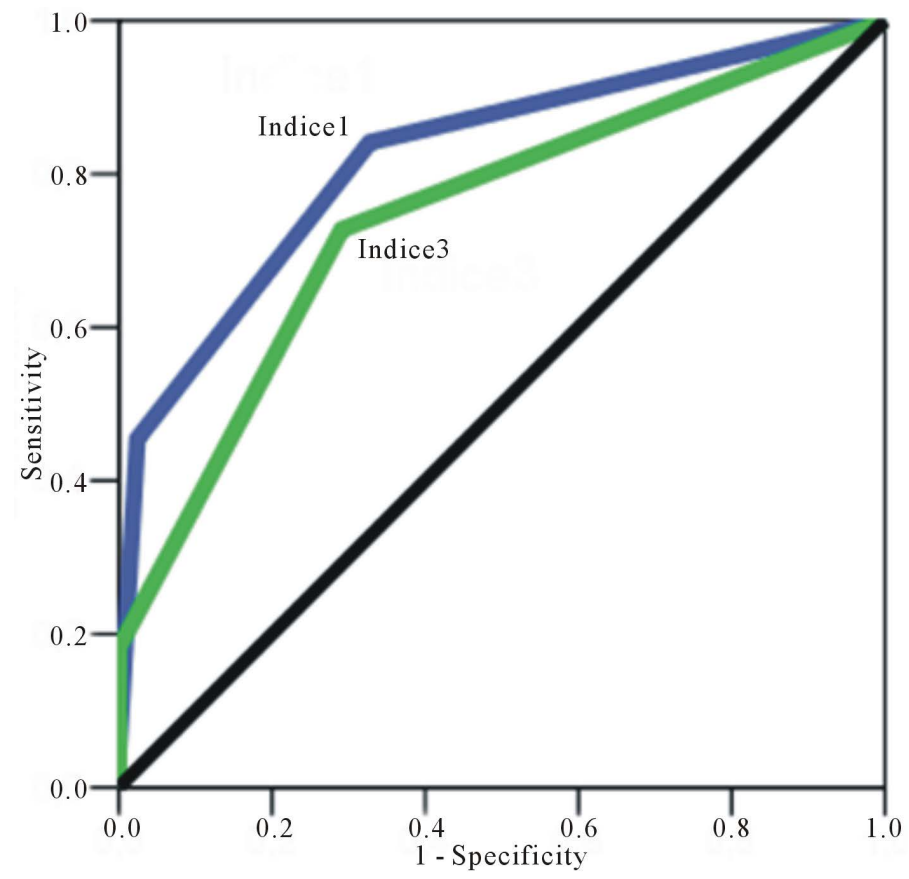

Figure 2. ROC curves for socioeconomic indice1 and indice3 (from simple addition score).

tion for the children. The used social indices express HIV impact on individuals and households. In the family, parent's death lead to orphan situation and must be considered in ART initiation. For the socioeconomic status, it has been reported that HIV affects more adults with better socioeconomic status [12] [13].

Other variables could be used to estimate the risk of severely malnourished children to be HIV infected or not: positive HIV status of his parents; long course infectious disease of parents, including brotherhood. However, 


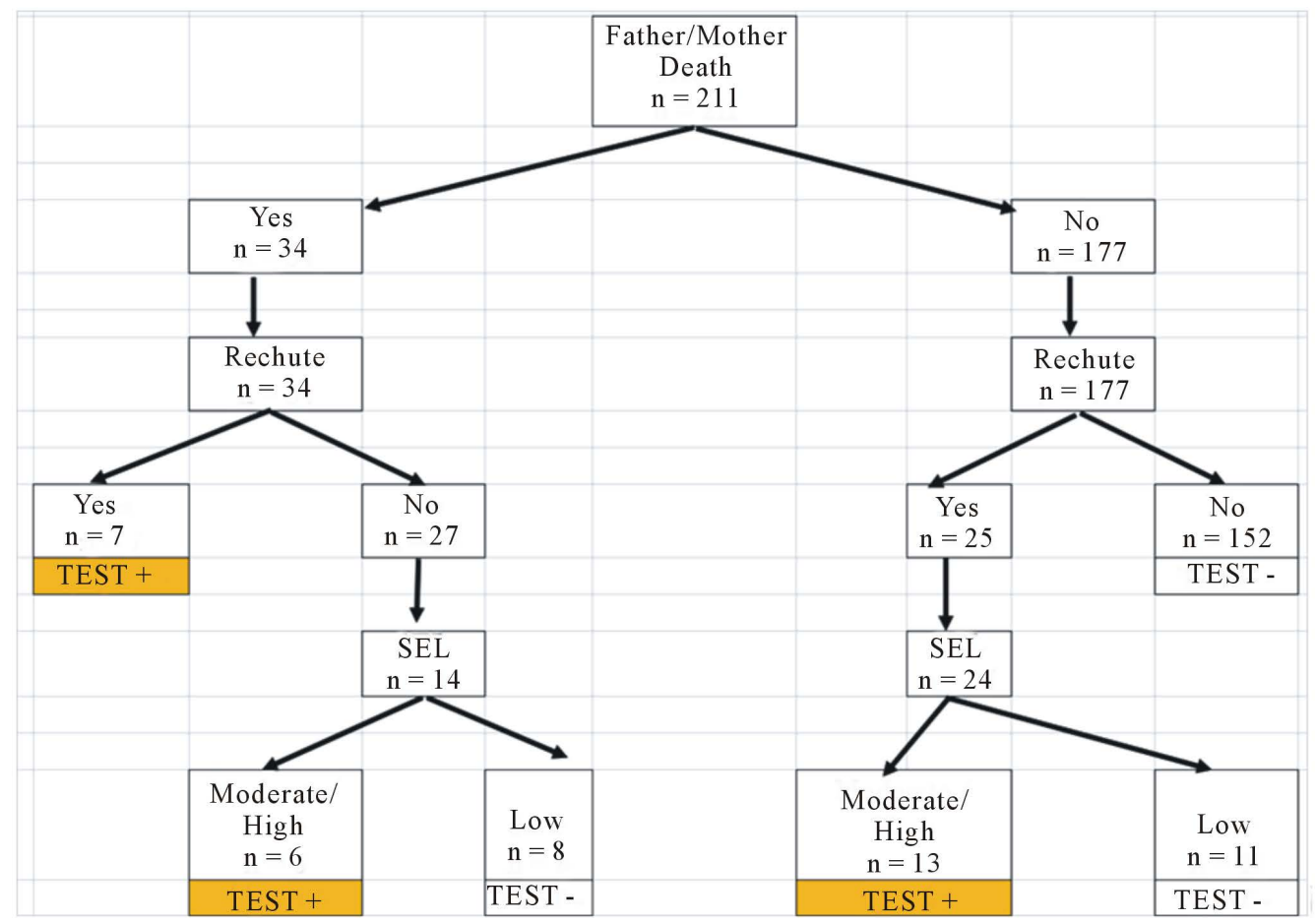

Figure 3. Classification of severely malnourished children at admission as positive (HIV infected) or negative (HIV uninfected) using Socioeconomic indices. Sensitivity (Se), Specificity (Sp), Positive Predictive Value (PPV) and Negative Predictive value (NPV).

experience shows that all these relevant information's are not readily obtained at the first medical contact.

HIV diagnosis testing is the first step for ART. It is essential to know context under which this ARV will be done. Using a performed socioeconomic indices scheme offers this advantage and help to focus on severely malnourished children at most risk to be HIV infected.

\section{Conclusion}

With appropriate cutoff, socioeconomic indices showed high specificity to discriminate, at admission, severely malnourished HIV infected to uninfected children. The medical decision aid scheme to purpose strongly HIV diagnosis to severely malnourished children at admission, built from socioeconomic indices, showed high specificity and high positive and negative predictive values. This approach can help for expanding ART for children in low income countries and achieve the maximum ART benefits in child socioeconomic kneed context.

\section{Acknowledgements}

Commission universitaire pour le développement (CUD), Belgique.

\section{References}

[1] United Nations (WHO/UNICEF/UNAIDS) (2013) Global AIDS Response Progress Reporting. Department of Economic and Social Affair, Population Division 2013, World Population Prospects: The 2012 Revision.

[2] WHO (2013) Global Update on HIV Treatment 2013: Results, Impact and Opportunities: WHO Report in Partnership with UNICEF and UNAIDS. WHO, Genevia.

[3] Puoane, T., Sanders, D., Chopra, M., Ashworth, A., Strasser, S., McCoy, D., Zulu, B., Matinise, N. and Mdingazwe, N. (2001) Evaluating the Clinical Management of Severely Malnourished Children-A Study of Two Rural District Hospitals. South African Medical Journal, 91, 137-141.

[4] Deen, J.L., Funk, M., Guevara, V.C., Saloojee, H., Doe, J.Y., Palmer, A. and Weber, M.W. (2003) Implementation of WHO Guidelines on Management of Severe Malnutrition in Hospitals in Africa. Bulletin of the World Health Organi- 
zation, 81, 237-243.

[5] Kessler, L., Daley, H., Malenga, G. and Graham, S. (2000) The Impact of the Human Immunodeficience virus 1 on the Management of Severe Malnutrition in Malawi. Annals of Tropical Paediatrics, 20, 50-56. http://dx.doi.org/10.1080/02724930092075

[6] Chintu, C., Luo, C., Bhat, G., DuPont, H.L., Mwansa Salamu, P., Kabika, M. and Zumla, A. (1995) Impact of the Human Immunodeficiency virus Type 1 on Common Pediatric Illnesses in Zambia. Journal of Tropical Pediatrics, 41, 348-353. http://dx.doi.org/10.1093/tropej/41.6.348

[7] Ndekha, M.J., Manary, M.J., Ashorn, P. and Briend, A. (2005) Home-Based Therapy with Ready-to-Use Therapeutic Food Is of Benefit to Malnourished HIV-Infected Malawian Children. Acta Paediatrica, 94, 222-225. http://dx.doi.org/10.1111/j.1651-2227.2005.tb01895.x

[8] WHO (2010) WHO Recommendations on the Diagnosis of HIV Infection in Infants and Children.

[9] Sombié, I., Nacro, B., Tiendrébéogo, S., Dao, B., Cartoux, M., Meda, N., Ky-Zerbo, O., Dabis, F., Mandelbrot, L. and Van de Perre, P. (1999) Maternal HIV Infection and the Anthropometric Characteristics of Children at Birth in Burkina Faso. DITRAME Study Group. Sante, 9, 173-177.

[10] Lepage, P., Msellati, P., Hitimana, D.G., Bazubagira, A., Van Goethem, C., Simonon, A., Karita, E., Dequae-Merchadou, L., Van de Perre, P. and Dabis, F. (1996) Growth of Human Immunodeficiency Type 1-Infected and Uninfected Children: A Prospective Cohort Study in Kigali, Rwanda, 1988 to 1993. The Pediatric Infectious Disease Journal, 15, 479-485. http://dx.doi.org/10.1097/00006454-199606000-00003

[11] Beau, J.P. and Imboua-Coulibaly, L. (1997) Caractéristiques antrhopométriques d'enfants malnutris infectés par le VIH en Côte d'ivoire. Cahiers Santé, 7, 236-238.

[12] Bollinger, L., Stover, J. and Zongo, J. (1999) The Economic Impact of AIDS in Burkina Faso. Research Triangle Institute (RTI)/The Centre for Development and Population Activities (CEDPA).

[13] Commision Economique pour l'Afrique (2004) Commission du VIH/SIDA et de la Gouvernance 2004. Le VIH/SIDA et le monde du travail. http://www.uneca.org 